\title{
Novel model for the simulation of lightning stepped leader
}

\author{
Udaya Kumar and G.R.Nagabhushana
}

\begin{abstract}
The stepped leader forms the first phase of the first stroke in any lightning flash. It is basically a stepwise elongating discharge in air, which carries significant charge. The charge distribution on the leader and its velocity of descent determine the attractive radius in shielding efficiency calculations. Also, the conductivity and the charge distribution on the leader tip region govern the initial stages of the return stroke. Therefore, there is a need for determining these parameters accurately. In this regard, a self-consistentmodel for the stepped leader will be very useful. The authors present a new model for the stepped leader that does not assume basic quantities like leader charge, current and velocity, but derives them from the computer simulation.
\end{abstract}

\section{Introduction}

All cloud-to-ground lightning flashes are preceded by a stepped leader phase $[1,2]$. The stepped leader, being a precursor to the first return stroke, is a stepwise elongating electrical discharge in air originating either at the cloud base or sometimes from tall objects (standing above ground). Statistics associated with the lightning flash can be found in $[1,2]$. As the magnitude and rate of rise of currents associated with the return strokes are much higher than that of the leader, these have been of main concern in the modelling of lightning. However, it may be noted that the return stroke neutralises the charge contained in the stepped leader and therefore it is important to assess the charge distribution on the leader. Earlier works on the computation of the return stroke current have modelled the stepped leader at its last stage as a finitely conducting cylindrical structure surrounded by a corona sheath [3-7]. The charge on the leader was either assumed to be constant [7] or determined by either assuming a constant potential all along the leader length [4-6] or by assuming a uniform gradient throughout [3].

The other major area concerned with the stepped leader is in the calculation of attractive radius/shielding efficiency of lightning protection structures. In any lightning protection system, it is required that the connecting streamers from the protective system should establish contact with the descending stepped leader before that from the system to be protected. The length of this connecting streamer for any system is mainly determined by the charge distribution on the tip region of the stepped leader and the actual velocity of its descent. In the literature pertaining to attractive radius/shielding efficiency calculations, the stepped leader has been modelled as a continuously descending cylindrical-conical charge structure with a charge distribution either assumed to be stepwise constant [8] or to be varying linearly with the peak at its tip $[9,10]$. Collectively, it may be observed here that in both of the aforementioned works, the leader needs to be considered at its final stage, and therefore no attempts have been made to model the leader from its initiation. In those cases seeking to explain the measured slow electric field-change records on the ground, the stepped leader has been considered as a continuously descending cylindrical charge structure [11].

In conclusion, it may be observed that much thought has not been put into the modelling of the stepped leader from its initiation and to describing its stepwise propagation. This work presents a novel model for the stepped leader which does not assume basic quantities like leader charge, potential, current, velocity and stepwise propagation characteristics, but derives them from the simulation.

\section{Proposed model}

Modelling of the stepped leader can be divided into:

(a) modelling of the air breakdown process, which is very active near the tip

(b) computations of the associated transient electric field that is the main cause of leader progression.

The assumptions made for minimising the computational burden are:

(i) The leader and the streamer are cylindrical in structure and they ascend or descend vertically straight. (This assumption is common to many of the earlier works.)

(ii) As the phenomena behind the initiation of the leader is not very clearly known [2], an initial leader of small length is always assumed. This will obviate the necessity for a high gradient at the cloud charge centre.

(iii) The development of the leader is similar to that during breakdown of long air gaps under switching impulses and therefore the model used there, with some modifications, can be extended to the leader.

(iv) Earth is modelled as a homogeneous, linear, lossy dielectric medium. This enables the use of the methodology of generalised images [12]. 
(v) Considering the potential of the cloud charge centre (from which the leader originates), the corona sheath radius of the leader is fixed. Charges in the cloud are approximated to either spherical,or cylindrical charge distributions (symmetric with the axis of the leader).

In the following, modelling of the individual phases will be discussed in detail.

\section{I Modelling of the air breakdown process}

Similarity between the leader development in natural lightning and that in long air gaps subjected to switching surges has long been observed [13]. The models employed for the analysis of switching surge breakdown in long air gaps therefore could be extended to model the lightning stepped leader. Owing to the complexity of the associated phenomena, only the macroscopic models, which explicitly do not refer to any of the microscopic phenomena, would be manageable. The leader progression model presented by Hutzler and Hutzler-Barre [14] is one such simple model, which does not refer to any of the ionisation process explicitly. However, both charge and step length of the leader are to be taken from the experimental data. Rizk [15] has developed a continuous leader inception model and has applied it to model the lightning incident on tall structures. Also in his work, the charge and velocities were taken from the measured data and only a continuously growing leader was considered. A stepwise growing lightning leader suggests a temporal growth of electric field and therefore the charge on the newly formed leader portion. For a realistic simulation of the leader, this charge growth has to be accurately traced through the associated governing fields, whereas all the earlier works have fixed a constant charge density all along the leader. The present work, while retaining the philosophies of the leader progression model for the inception fields, fields inside the leader and for the streamer length, makes no assumptions regarding charge, current and velocity of the leader. These quantities will be deduced through the simulation. Modelling of the air breakdown process will now be discussed.

The leader is considered as an electric arc comprising a thin (radius $<2 \mathrm{~cm}$ ) but highly conducting central core, which is surrounded by a corona sheath (radius in metres). Most of the conduction is limited to the central core, whereas most of the charges are held in corona sheath. Whenever the gradient at the tip of the leader exceeds the local value of the critical gradient $E_{c r}(h)$, a streamer having a constant gradient $E_{s t}(h)$ is assumed to be formed [14]. The length of the streamer is fixed by selecting a point on the axis where the average gradient is equal to the minimum streamer propagation gradient [15]. Furthermore, streamer conductivity needs to be determined which dictates, with the streamer gradient, the current inside. For the streamer conductivity, a simplified Braginskii's formulation [16] is employed, which can be expressed as

$$
r\left(t_{1}\right)=r\left(t_{0}\right) \frac{\int_{0}^{t_{0}} i(t)^{2 / 3} d t}{\int_{0}^{t_{1}} i(t)^{2 / 3} d t}
$$

where $r(t)$ is the resistance of the streamer segment under consideration, $i(t)$ is the current through the segment and $t_{0}, t_{1}$ are two consecutive time instants. The initial value for $r(0)$ is taken as $100 \mathrm{k} \Omega / \mathrm{m}$. occurred. For the conductivity of the leader, the generalised Mayr's equation $[14,15]$ is employed

$$
\frac{d g}{\left(g_{\infty}-g(t)\right)}=\frac{d t}{\theta}
$$

Where $g(t)$ is the conductivity at any instant $t, \theta$ is the time constant and is taken as 50 $\mu$ s [10] and $g_{\infty}(i)=(A i(1+\alpha))^{-1}$ $=\left(0.75 \times 10^{5} i^{-15}\right)^{-1}$. With this type of formulation for the conductance of the leader, no explicit reference either to actual radius or to the conductivity of the central core becomes necessary. As these are complex functions of current and time, they are very difficult to obtain directly. To limit the current, it was necessary to put a lower limit on the leader resistance and this was taken arbitrarily as $20 \Omega / \mathrm{m}$. The current flowing through the nonlinearly conducting leader and streamer channel builds up the charge and hence the potential of the leader tips. When it exceeds the critical gradient $E_{c}$, a new step will be formed.

The values of the various breakdown parameters are as follows. The critical gradient at STP $(26 \mathrm{kV} / \mathrm{cm}$ as given in $[17])$ is taken as the boundary of the active region. Thum et al. [9], also employ a similar value. The positive streamer gradient is taken as $4 \mathrm{kV} / \mathrm{cm}$ in the works of Hutzler and Hutzler-Barre [14] and Rizk [15]. This seems to be a well established value and has been used in the present work. However, the data on negative streamer gradient is not so well established. Ohta et al. [18], using Pockel's device, have experimentally measured the gradients in positive and negative streamers and also in leaders. The measured gradients are $5-14 \mathrm{kV} / \mathrm{cm}$ for the positive streamer, $18-32 \mathrm{kV} / \mathrm{cm}$ for the negative streamer and $1.5-5.5 \mathrm{kV} / \mathrm{cm}$ for the leader. Even the minimum positive streamer gradient measured by Ohta et al. [18] is more than the widely employed value. This can be attributed to the lower values of the leader currents encountered in the work of Ohta et al. [18]. Considering this, the measured negative streamer gradient of Ohta et al. [18] is scaled with the ratio of the established positive streamer gradient to the measured gradient and then the negative streamer gradient is taken as $14.4 \mathrm{kV} / \mathrm{cm}$. Considering the complexity of the problem and lack of credible data, the effects of rain, mist and humidity on the streamer and leader gradients are not considered. Allen and Ghaff [19] have shown that for temperatures in the range $-14^{\circ} \mathrm{C}$ to $+148^{\circ} \mathrm{C}$ and for air density in the range $0.6-1.1$, the air density and temperature correction factor is equal to the relative air density $\delta^{1.5}$. Considering the typical height of the negative charge centre in the cloud to be $5-6 \mathrm{~km}$, the air density encountered is greater than 0.6 and temperature is greater than $-24^{\circ} \mathrm{C}$. Therefore, the correction factor given by Allen and Ghaff [19], is very suitable for the present work and has been employed for both streamer gradients and the critical gradient. The resulting correction factor, up to a height of $5 \mathrm{~km}$, follows the relation

correction factor $=1.00514 \times \exp (-0.000187214 h)$

where $h$ is the height in metres above ground.

\subsection{Computation of the transient electric field}

Electric field build-up at the tip of the leader acts as the prime mover for the leader propagation. This field build-up is realised by the accumulation of the charges in the newly formed leader segments. Therefore, for an accurate trace of the tip electric field build-up, it is essential to follow the charge build-up phenomena. Researchers have postulated a capacitive charging phenomenon (for example, see Golde [20]), although no attempt has been made to compute it. Owing to the lower velocities of the stepped leader as com- 
pared to the velocity of light, the wave propagation and eddy current effects are negligible. Therefore, the electric field distribution can be described by Laplace's/Poisson's equation and the magnetic field by vector Poisson's equation. It has been shown in earlier work $[16,21]$ that the transient phenomenon arises solely because of the timedependent material boundary conditions to be satisfied by these fields. A detailed study of the field associated with the stepped leader reveals that it belongs to the resistiveinductive-capacitance (RLC) type of transient fields [16].

Modelling of the Earth plays a major role in the estimation of the electric field near ground and in the computation of the length of the connecting streamers. Owing to the wide range of Earth conductivity $(0.005-0.5 \mathrm{~S} / \mathrm{m})$ and permittivity $\left(3<\varepsilon_{r}<81\right)$ profiles, it can neither be modelled as a dielectric nor as a conductor for which classical image theory applies. In view of this, a general theory of images applicable to linear lossy dielectrics developed in an earlier work [12] has been employed.

\section{Numerical implementation}

The field associated with the stepped leader is not amenable to any simple analytical techniques and hence a numerical approach has to be adopted. The physical charges are directly discretised and are used in the evaluation of the electric fields. For the numerical computation, the leader poses a difficult field problem because of its very adverse aspect ratio (radius of a few metres and length of a few kilometres). In addition, the leader segments of few metres in diameter, carrying charges in the range of milli-coulombs, need to compete with the cloud charges of the magnitude of a few tens of coulombs and dimensions of hundreds of metres. Therefore, great care needs to be exercised in controlling the numerical errors. The number of time steps required for a final leader length of $2.5 \mathrm{~km}$ is of the order of $10^{6}$. Fixing an accuracy of $1 \%$ for the gradients inside the leader/streamer, the resulting accuracy requirement for the computed voltages would be of the order of $10^{-5}-10^{6 \%} \%$ [16]. Considering all these, spherical charge structures are used for the spatial discretisation of the streamer and the leader corona sheath. During the initial phases of leader/ streamer development cylindrical charge structures, when employed for the spatial discretisation, are found to perform very poorly (numerically). However, for the old matured leader segments, replacing many spherically discretised leader portions by a single cylindrical segment proves computationally very beneficial.

During the development of the leader, its resistance falls from $100 \mathrm{k} \Omega / \mathrm{m}$ to a value of $20 \Omega / \mathrm{m}$. The very poor aspect ratio of the problem together with this nonlinearity demand an extremely small time step, and therefore a very large number of computations. An improvement of more than a factor of ten can be realised by employing a nonlinear numerical filter [22] to the computed leader voltages. It suppresses the spatial oscillations (which could result in a blow-up) without violating conservation. This spatial oscillation was otherwise found to initiate at the root of the leader especially with larger time steps. The final time step after the incorporation of the above filter was $10^{-9} \mathrm{~s}$.

The numerical implementation of the streamer formation needs special attention. The streamer demands a fixed average gradient inside, which cannot be met by using charges as the charges cannot be moved instantaneously. Therefore, a fictitious polarisation of suitable magnitude was used [16]. This could also be viewed as a mimic to the number of avalanche heads (forming the streamer) containing charges of both the polarities effectively separated in space. The geometry used for this polarisation is obviously the same as that of the streamer. If $\boldsymbol{E}_{0}$ is the actual existing field and $\boldsymbol{E}_{s t}$ is the desired streamer gradient, then the amount of polarisation required is

$$
\boldsymbol{P}=\left(3 \varepsilon_{0} \frac{\left(\varepsilon_{r}-1\right)}{\left(\varepsilon_{r}+1\right)}\right) \boldsymbol{E}_{0}
$$

where $\varepsilon_{r}$ is given by

$$
\varepsilon_{r}=\left(3 \frac{\left|\boldsymbol{E}_{0}\right|}{\left|\boldsymbol{E}_{s t}\right|}-2\right)
$$

During the whole of the streamer phase the gradient inside is held constant at the streamer propagation field. This has been realised by decreasing the imposed fictitious polarisation with the charge accumulation in the streamer. When the charges accumulated in the streamer reach the level of fully meeting the gradient inside, the fictitious polarisation is removed. In addition, this stage also indicates the attainment of good conductivity. Therefore, the associated streamer segments are immediately converted into leader segments.

The electric potential all along the leader needs to be evaluated at each discretised time interval and the algorithm adopted is outlined as follows:

(a) from the known charge distribution compute the potential all along the leader segments employing:

(i) for spherical volume charge distribution

$$
\begin{aligned}
V(r) & =\frac{4}{4 \pi \varepsilon_{0} r} & r>R \\
& =q \frac{\left(R^{2}-r^{2}\right)}{8 \pi \varepsilon_{0} R^{3}}+\frac{q}{4 \pi \varepsilon_{0} R} & r \leq R
\end{aligned}
$$

(ii) for spherical volume polarisation (along the axis)

$$
\begin{aligned}
V(r) & =|\boldsymbol{P}| \frac{R^{3}}{3 \varepsilon_{0} r^{2}} & & r>R \\
& =|\boldsymbol{P}| \frac{r}{3 \varepsilon_{0}} & & r \leq R
\end{aligned}
$$

where $q$ is the total charge, $\boldsymbol{P}$ is the dipole moment per unit volume, $\boldsymbol{R}$ is the radius of the spherical volume and $r$ is the distance of point of measurement from the centre of the spherical volume.

(b) then compute the current distribution all along the leader following RLC type transient fields [21]

$$
\int_{\boldsymbol{A} \boldsymbol{I}} \rho(z, t) i(z, t) d l+\frac{\partial}{\partial t} \int_{\boldsymbol{A} \boldsymbol{I}} \boldsymbol{A} \cdot d l=\Delta V
$$

where $\mathbf{A V}$ is the voltage across any segment of length 1 , $\rho(z, t), A(z, t)$ and $i(z, t)$ are the resistivity, divergence free magnetic vector potential and current profiles along the leader, respectively. The arguments $(z, t)$ denote the spatial and time variables. Considering the weak coupling between two adjacent leader segments, all the mutual couplings can be safely neglected. As a result, only the self-coupling terms remain and further by assuming a constant (or average) current throughout the segment, magnetic linkages can be deduced in terms of the familiar self-inductance $L_{l}$ of the segment. The expression for $L l$ is [23]

$$
L_{l}=0.002 l\left(\ln \left(\frac{2 l}{r_{\text {core }}}\right)-0.75\right)
$$

where $l$ is the length of the leader segment under consideration and $r_{\text {core }}$ is the radius of the leader core $(1-2 \mathrm{~mm}$ to $\mathrm{Icm})$. In eqn. 8 , both the length and radius are in $\mathrm{cm}$ and 
inductance is in $\mu \mathrm{H}$. With this, eqn. 8 can be reduced to

$$
R_{l} i(z, t)+L_{l} \frac{\partial i(z, t)}{a t}=\Delta V
$$

where $R_{l}$ represents the average resistance of the segment at the time instant $t$.

(c) from the spatial current data pertaining to any time instant $t$, the charge accumulation can be determined according to the theory of RC-type field transients [21, 24]

$$
\frac{\partial i(z, t)}{\partial z}=-\frac{\partial \lambda(z, t)}{\partial t}
$$

where $\lambda(z, t)$ represents the linear charge density from which the equivalent spherical volume charge can be computed. As mentioned earlier, both conductivity and permittivity of the Earth participate in determining the image of charge distribution in air. According to the generalised theory of images, the equivalent set of images would be dielectric image plus the equivalent charge for surface charges, which are being built-up. For any time-varying point charge $q(t)$, (representing the uniform spherical volume charge distribution for the present case) in air, the equivalent image charge $q_{s}$ for the accumulating surface charge distribution is given by [12]

$$
\begin{aligned}
& \frac{\partial q_{s}}{\partial t}+\left(\frac{\sigma_{\text {earth }}}{\varepsilon_{\text {air }}+\varepsilon_{\text {earth }}}\right) q_{s} \\
&=\frac{2 \varepsilon_{\text {air }}}{\left.+\varepsilon_{\text {earth }}\right)}\left\{\frac{-\sigma_{\text {earth }}}{\left(\varepsilon_{\text {air }}+\varepsilon_{\text {earth }}\right)}\right\} q(t)
\end{aligned}
$$

The equivalent charge $q_{s}$, is to be kept at the image point and is valid for all the calculation in air.

(d) increment the time, go to step 1 after checking for the critical field at the leader tips and evaluating new values of conductivities.

\section{Data used for the simulations}

A common model for the cloud charges employed in the literature comprises a positive charge centre at the top, which is followed by a negative charge centre at the bottom. This negative charge has been considered in the present work to be in two volumes and out of which only the bottom negative charge volume will be electrically connected with the leader. Due to the assumptions made, cloud charges are aligned one below the other along the axis of the leader. Unless otherwise stated, the values quoted in Tables 1-3 were used in the simulation. Further, the arc constants and other geometrical details used in the simulation are as follows:

arc constants: $\boldsymbol{A}=0.75 \times 10^{5}, a=0.5$, time constant $\theta=$ $50 \mu \mathrm{s}$

length ahead of the leader prescribed for evaluating the averaged tip gradient to be used as the critical gradient $l_{x^{\prime} \text { ad }}$ $=r_{C}$

length of the preliminary leader $=40 r_{c}$

minimum length of the spatial discretisation used $2 r_{c}=$ $16 \mathrm{~m}$

radius of the leader corona shell $r_{l c}=r_{c} / 1.225$

all cloud charges were represented by spherical charge distributions.

A detailed sensitivity analysis has been carried out to ascertain the influence of various parameters on the performance of the model. This was required to ensure that the possible variation in these parameters does not invali- date the model. Results of the study show that the proposed model is not very sensitive to its parameters [16].

Table 1: Details of the cloud charge distribution

\begin{tabular}{lll}
\hline Charge type & Height, km & $\begin{array}{l}\text { Charge } \\
\text { magnitude, C }\end{array}$ \\
\hline Positive (at the top) & $Z_{p}=14$ & $Q_{p}=30$ \\
Negative (atthe bottom) & $Z_{n}=5.5$ & $Q_{n}=-25$ \\
Participating negative & $Z_{a}=3.0$ & $Q_{a}=-5$ \\
\hline
\end{tabular}

Table 2: Various electric field gradients used for the simulation

\begin{tabular}{ll}
\hline Critical gradients & Value at STP, $\mathrm{kV} / \mathrm{cm}$ \\
\hline Breakdown $\left(\mathrm{E}_{\mathrm{cr}}\right)$ & 26 \\
Positive streamer propagation & 4 \\
Negative streamer propagation & 14.4 \\
\hline
\end{tabular}

Table 3: Initial resistances used for the simulation

\begin{tabular}{ll}
\hline Streamer & $100 \mathrm{k} \Omega / \mathrm{m}$ \\
Preliminarv leader & $1 \mathrm{k} \Omega / \mathrm{m}$ \\
\hline
\end{tabular}

In the following, results obtained from the simulation are discussed with reference to step length and duration, leader charge, leader current, leader voltage profile, leader tip voltage and field. Also, the potentials and fields at selected points on the earth are presented. For brevity, only the important results of the simulation are discussed. For general information, a few details of the computation facility used are as follows:

The computer code was written in $C$ language and the computations were carried out in SUN-Sparc2 and IBM workstations. Typical run times are of the order of a few hundred minutes to a few thousand minutes of CPU time (depending on the radius of the corona sheath and the height of the participating charge centre from the ground).

\section{Simulation results and discussions}

\section{I Step length and duration}

Results of the simulation indicate that the step length remains constant for a given leader radius and charge polarity. It may be recalled that the simulation was carried out only for a vertically straight leader without any branches. Step lengths are much larger for positive leaders compared to negative leaders and the ratio of positive to negative leader step lengths approximately follows the ratio of propagation gradients of positive and negative streamers. Under identical conditions the step length and average step duration for a positive leader are $192 \mathrm{~m}$ and $200 \mu$ s and the respective values for the negative leader are $48 \mathrm{~m}$ and 48.6 $\mu$ s. The step duration, however, varies with the leader length and potential/field near the participating charge centre. Initially, due to the preliminary leader, the prevailing conditions are different from those at the later stages where the leader propagates naturally. Therefore, there exists an oscillation in the initial period. Later, until the leader comes close to the ground, the step duration increases with leader length. However, near the ground, step duration reduces, as shown in Fig. 1. Simulations with different values of streamer propagation gradients indicate that the larger the step length, the larger the step duration as observed in practice. Table 4 shows the influence of the participating cloud charge magnitude on the leader step duration and total charge injected. 


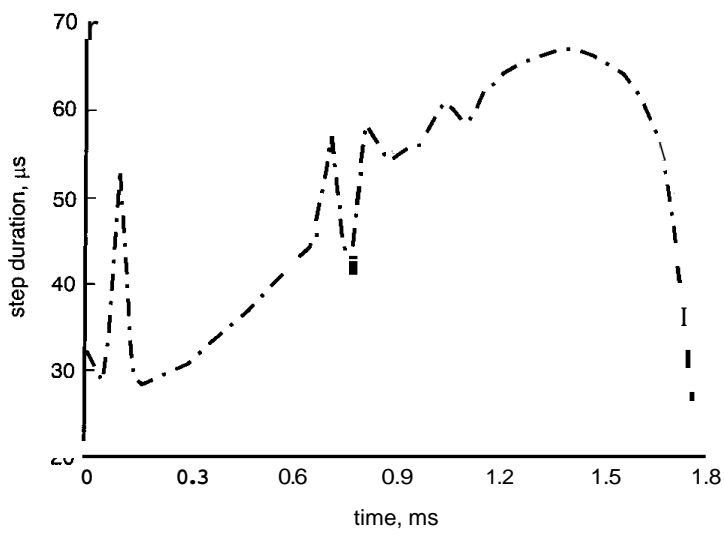

Fig.1 Typical variation of step duration

Table 4 Influence of participating cloud charge magnitude on leader propagation

\begin{tabular}{llllll}
\hline$\alpha_{a}$, C & $\begin{array}{l}\text { Cloud } \\
\text { potential, } \\
\text { MV }\end{array}$ & $\begin{array}{l}\text { Step } \\
\text { length, } \\
\mathrm{m}\end{array}$ & $\begin{array}{l}\text { Total leader } \\
\text { duration, } \\
\text { rns }\end{array}$ & $\begin{array}{l}\text { Avg. step } \\
\text { duration, } \\
\mu \mathrm{s}\end{array}$ & $\begin{array}{l}\text { Total } \\
\text { charge, C }\end{array}$ \\
\hline 5 & 167 & 48 & 1.76 & 48.63 & 1.38 \\
6 & 189 & 48 & 1.03 & 28.50 & 1.34 \\
7 & 212 & 48 & 0.77 & 21.51 & 1.54 \\
8 & 234 & 48 & 0.62 & 17.32 & 1.63 \\
\hline
\end{tabular}

It may be observed that the step length and the average step duration for the negative leader match favourably the well established (measured) values of $50 \mathrm{~m}$ and $25-50 \mu \mathrm{s}$, respectively. The computed leader velocities are close to or slightly higher than the maximum value of the measured leader velocities. However, it should be noted here that the measured leader velocities are only the two-dimensional velocities and, in reality, the stepped leader follows a very tortuous path. Therefore, the actual velocity of the stepped leader will be higher than the two-dimensional velocity computed from lightning photographies.

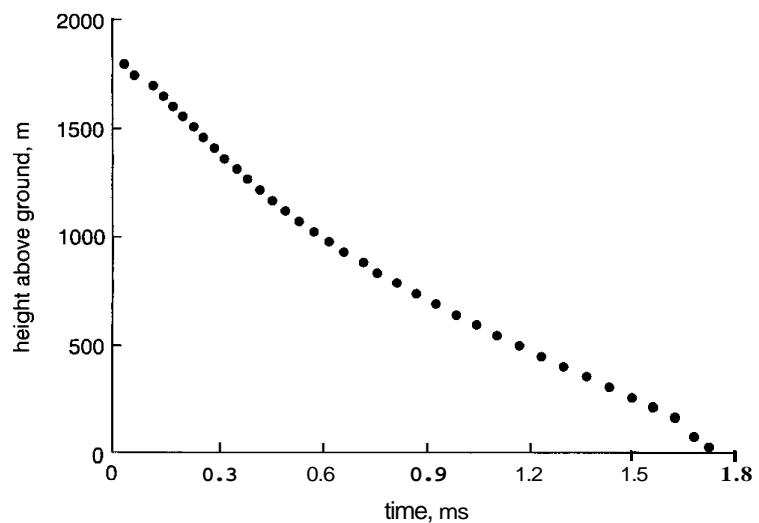

Fig.2 Locus o the descending leader tip

Fig. 2 shows the height of the leader tip as a function of time. To start with there will be a preliminary leader, and then the tip descends at a constant rate. At about half the distance, the speed of descent decreases as also indicated by the step duration plot in Fig. 1. Closer to the ground, over the last few steps of descent, the speed increases sharply. Initially, when the leader lengths are small, the total leader resistance will also be small. In addition, it has strong elec- trostatic coupling only with the cloud charges. Thus, charge build-up, and consequently the build-up of tip potential and tip gradient, will be faster. Later, when the leader has covered nearly $50 \%$ of the gap, its resistance would also have increased and the electrostatic coupling now will be with both cloud charges and the Earth. Therefore, more time is required for the charge build-up and hence the tip gradient. However, when the leader comes closer to earth, due to the stronger influence of the image charges in the earth, build-up of the gradient is much faster. This geometry-dominated charge and field build-up will overwhelm the increase in total leader resistance, thus resulting in shorter step durations.

\subsection{Leader charge}

Fig. 3 shows the typical profile of computed charge density along the leader for different leader lengths. It may be observed that the leader charge density remains low at the leader root and then increases sharply. But, for larger leader lengths, this sharp increase will disappear within $25-50 \%$ of the leader length and a moderate increase will be found. At the tip, the charge density (and charge) builds up sharply after a step. The charge density is lower at the leader root as the field in that region is strongly governed by the cloud charges. Later, the charge in the leader also contributes significantly to its potential, which is high, and hence the charge density increases. In the region near the tip, as in the case of the conductor tip, the charge density increases sharply (but is limited by the formation of a new step). It may be recalled that in earlier works, leader charge distribution was either assumed to be piecewise constant [8] or to increase linearly from the root to the tip $[9,10]$

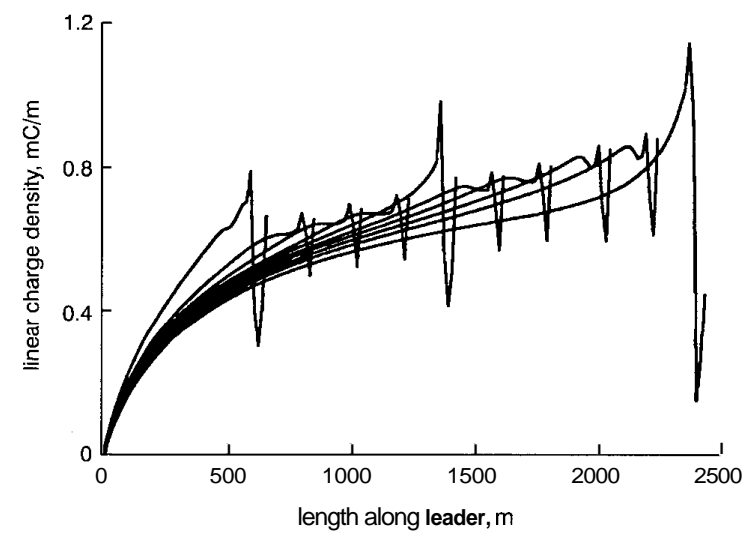

Fig.3 Leader charge profile at different lengths

Fig. 4 shows the total charge on the leader as a function of time. It may be noted that the charge injection to the leader is a smooth process. It may be recalled that many researchers [11, 25], to explain far-distance electric measurements, have modelled the leader as a vertically straight, uniformly charged cylindrical volume descending at a uniform velocity. At far distances (i.e. distances more than the total length of the leader itself), the fine structure of the leader charge distribution becomes nearly invisible and only a gross effect will be seen. Both Figs. 2 and 4 indicate almost a steady increase in leader length and its total charge, thus justifying the approximation of the above researchers. However, for the attractive radius calculations and for determining the initial conditions relevant to return stroke computations, near fields are important. Therefore, the fine structure of the leader charge (and conductivity) is very 
essential. It is observed from the results of simulation that the computed total leader charges are always lower (by $45 \%$ or less) compared to the estimates reported in the literature. This can be expected as only a vertically straight descending leader is simulated, whereas, in reality the leader exhibits extensive branching and follows a tortuous path. Therefore, the total leader length in natural lightning will be more than the height of its root above ground. When the length is more, electrostatic couplings will be higher as also will be the total charge accumulated.

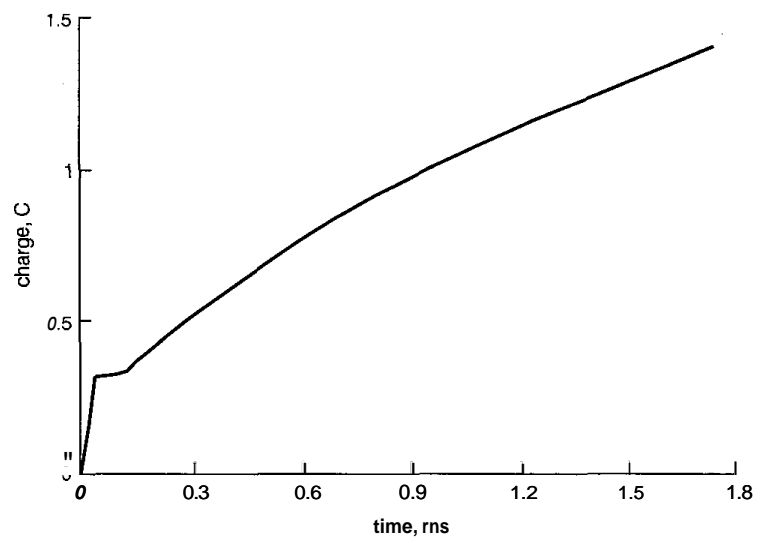

Fig.4 Temporal growth of total charge on the leader

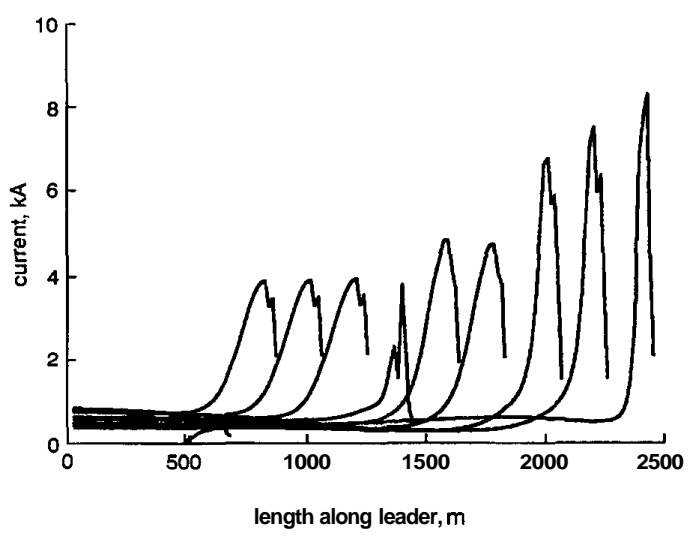

Fig.5 Leader current profile at different lengths

\subsection{Leader current}

The leader current is not a well established quantity as direct measurements on a stepped leader are not practical. However, by knowing the leader transit time and the total leader charge, the leader current was deduced to be a few hundred amperes. Values of $100-300 \mathrm{~A}$ are frequently referred to as typical (average) values [1, 10]. But careful measurement of high-frequency magnetic field pulses due to the leader by Baum [26] has led to the estimation of leader currents to be at least $1 \mathrm{kA}$ and range up to a few tens of kA in new leader steps. Simulation results clearly confirm this aspect. The older leader segments carry currents in the range $300-600 \mathrm{~A}$. This current seems to decrease slightly along the length and at the new leader segments the current increases to the level of a few kiloamperes as shown in Fig. 5. At the latest leader segment, sharp current pulses of magnitudes up to a few tens of kiloamperes are also observed. These current pulses have very short rise and fall times. Baum [26], from his field measurements, concludes that the current in the tip seems to have rise times of the order of a few tens of nanoseconds. One- to-one comparison of the current magnitude and rise time is not possible as only a coarse discretisation is adopted in the present simulation. This was very necessary to limit the already huge computational burden. The coarse spatial discretisation has resulted in sharp fluctuation of the current at the tip. The plots in Figs. 3 and 5 are taken at equal space intervals. This does not correspond to the same time intervals and consequently to the same electrical conditions of the leader. At each length, the propagation stops for a time interval of step duration and severe electrical activities take place in the newly formed portion. As a result, plots contain dissimilar curves. It should be noted that the plots taken under the same electrical conditions give similar curves and this is avoided in the above to indicate that the curves show slightly different patterns at different times. The common observation from the results of the simulation is that the spatial current profiles show more peaking as the leader approaches the ground.

\subsection{Currentat various heights}

Currents at various heights can give further insight into the phenomenon. In Fig. 6, a leader current at $50 \%$ height is given. The currents measured at various heights show the initial pulsating nature of the leader current. The current rises sharply to a magnitude of the order of $10 \mathrm{kA}$ with the formation of the step. Owing to the coarse discretisation adopted in the simulation, occasional oscillations can be seen in the computed current magnitudes. Subsequently, this current falls to a few hundred amperes till the next step is formed; again the current rises generally to less than $50 \%$ of the previous peak. However, due to a coarse discretisation employed in the present work, sometimes it is found that the second peak in the current can exceed the first (as shown in Fig. 6). For the subsequent steps, this pulse peak diminishes more or less uniformly to become a ripple and then finally settles down to a uniform current. The pulsating nature of the initial current of a newly formed step is supported by the magnetic field measurements of Baurn [26]. Also, the photographs of the stepped leader show extensive brightness in each step near the tip where both the current and the gradients are high.

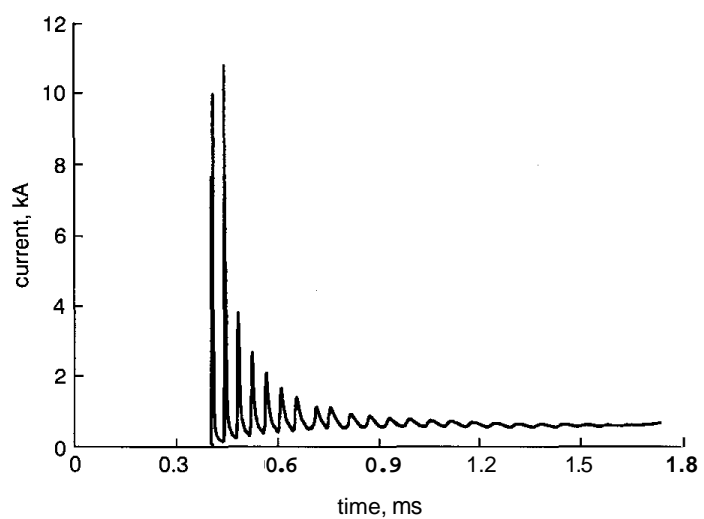

Fig.6 Leader current at $50 \%$ height above the ground

\subsection{Leader voltage profile}

Leader potentials at various leader lengths are shown in Fig. 7. These data are for an arbitrary instant and therefore correspond to different leader tip conditions. It may be observed from Fig. 7 that the leader gradient, which is dynamic in nature, remains constant except near the tip. Near the tip of the leader, which comprises the recent two or three steps, the potential of the leader drops sharply. 


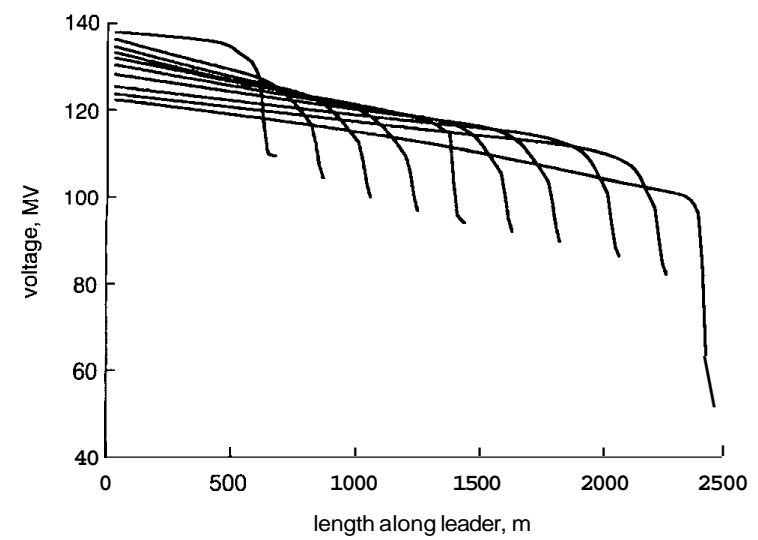

Fig.7 Potential along the leader at different lengths

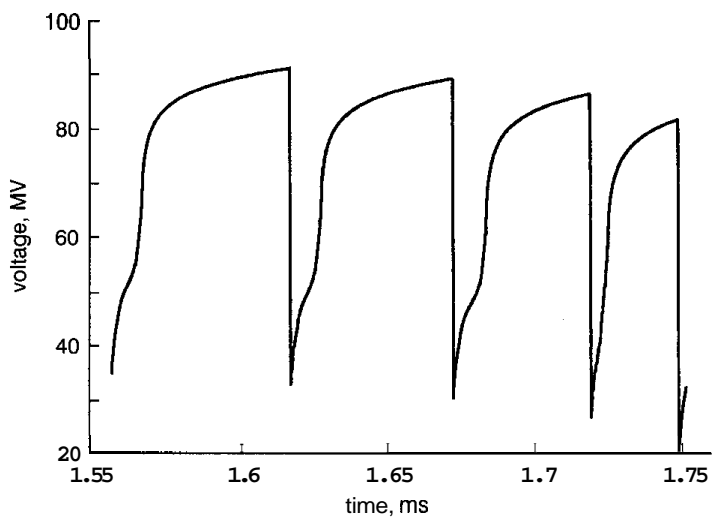

Fig.8 Leader tip voltage as afiction of time

\subsection{Leader tip voltage}

It will be interesting to study the leader tip potential, as the dynamic changes of the tip gradient would be indicated by it. For the sake of brevity and for better clarity, only a small interval spanning only the last few steps are shown in Fig. 8. It may be noted that the leader tip is not a futed point in space but will be moving (in steps), and therefore the leader tip potential is the potential of a point that is not futed in space. When a new step is formed the leader tip potential falls which is determined by the streamer gradient and initial leader tip potential. As the conductivity of the newly formed streamer improves (nonlinearly with the current), charge gets accumulated in it and the tip potential increases. This increase, as shown in Fig. 8, has two distinct phases. The first phase is due to the streamer and the second due to the leader phase. In the streamer phase, the potential is governed by both free charges and dipolar charge distributions. The change in conductivity is mainly governed by Braginskii's formulation. In the leader phase only charges determine the potential and the changes in conductivity are governed by Mayr's equation. The initial fast rise in potential is mainly due to the fast rise in conductivity. During this period, mainly local redistribution of the charges from the previous leader segments occurs. Later, due to the fall in gradient in this region, charge accumulation slows down and hence there is a rise in potential. When the potential of the tip reaches a magnitude that is sufficient to produce the critical gradient, a new step will be formed and the potential of the tip will drop. This is neatly demonstrated in Fig. 8.
5.7 Potential and electric field at the ground

During the simulation of the stepped leader, both the average potential and the average field (representing slow field records) near the ground at various radial distances were measured. It was observed from these measurements that both of them rise monotonically with leader progression. However, the fine resolution of the temporal potential shows kinks riding over an otherwise monotonically increasing trend. These kinks are formed due to the initial dielectric image of the charges in the new step which later changes into a conducting image. Fig. 9 shows the potential at a radial distance of $100 \mathrm{~m}$ from the leader axis at a height of $1 \mathrm{~m}$ above ground. The corresponding soil parameters are $\varepsilon_{r}=80$ and $\sigma=1.0 \mathrm{~S} / \mathrm{m}$ which represents seawater or a very highly conducting Earth. Fig. 10 shows the computed potential for two types of Earth. The first one corresponds to a highly conducting Earth (the same as above) and the second one corresponds to a poorly conducting Earth $\left(\varepsilon_{r}\right.$ is taken arbitrarily as six for dry Earth and $\sigma=0.006 \mathrm{~S} / \mathrm{m}$ ). It may be noted that the magnitude of the kinks is dependent on the soil conductivity and permittivities. Also, the width of the kinks is greater for lower soil conductivities. This is due to the higher time constants (i.e. $\varepsilon / \sigma$ ) associated with the lower soil conductivities. It should be noted that as the leader is far away from the ground, the effect of the steps is not felt significantly and it is only when it comes close to the ground that both potential and field show an initial sharp and then a slow increase with each step. Fig. 11 shows the electric field normal to ground surface measured at the ground surface at a radial distance of $100 \mathrm{~m}$ from the leader axis. The effect of the formation of each step and the corresponding salient changes in the temporal field can be observed in these plots (Figs. 9-1 1).

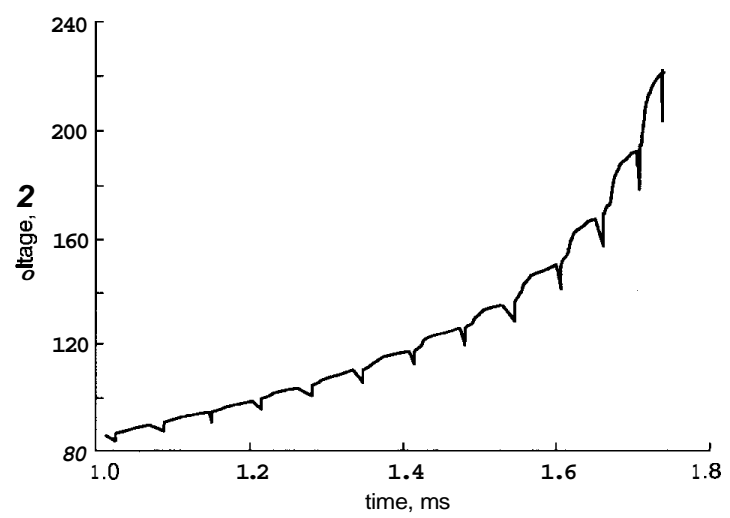

Fig.9 Variation $m$ the potential near the ground as the leader descends Highly conducting Earth, height $=1 \mathrm{~m}$, radial distance from leader $=10 \mathrm{~m}$

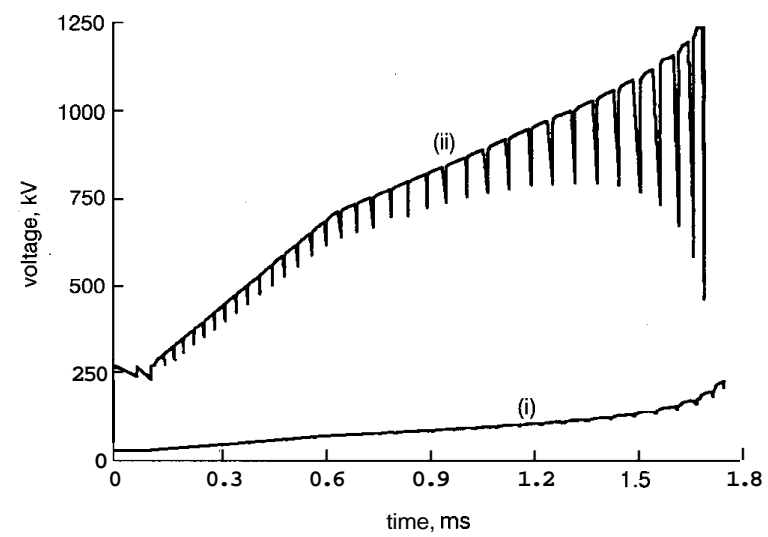

Fig,.10 Influence of earth conductivity on induced voltage near the ground (i) highly conducting Earth, (ii) poorly conducting Earth 


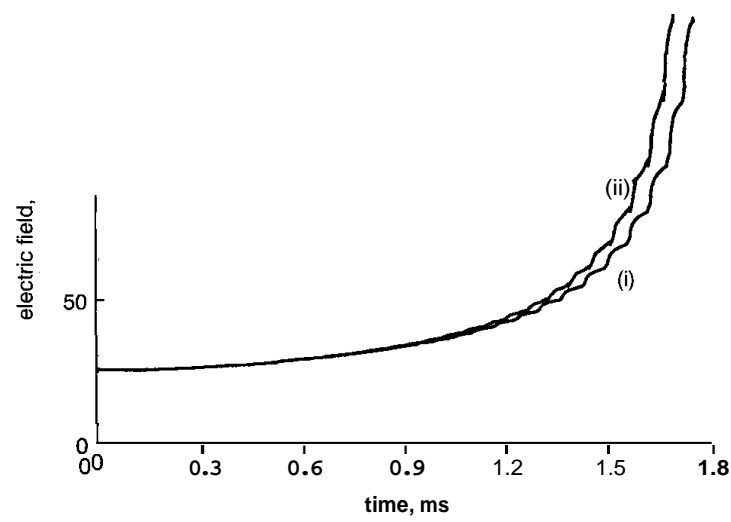

Fig.11 Normal component of electric field near the ground (i) highly conducting Earth, (ii) poorly conducting Earth

\section{Conclusions}

A new model for simulation of the stepped leader (of the first stroke) of natural lightning is presented. No assumptions are made regarding leader charge, current and velocity of propagation. Simulations have been carried out for a vertically descending straight leader. The computed step lengths and step durations are in good agreement with the field data for natural lightning, thus giving credibility to the model. The computed step lengths are larger for positive leaders than for negative leaders. It is also observed from the simulations that the longer the step length, the longer the duration, as in natural lightning. The leader currents and charge distributions in natural lightning are not very accurately known. From the high-frequency magnetic field measurements made at ground level and from the data pertaining to total leader charge and duration, leader currents are estimated to be a few hundred amperes along the length rising to a few to a few tens of kilo-amperes near the tip. The simulation results using the present model clearly confirm this aspect. Also, the leader voltage gradients and currents are found to be high near the tip supporting the photographic data of stepped leaders which show a relatively high intensity of light emission only near the tips. The computed leader tip voltage clearly indicates the cause of stepwise propagation. The Earth has been modelled more realistically as a linearly conducting dielectric and includes the electrical parameters of the soil and is found to influence the ground field. As observed in practice and predicted by the previous simpler models, the computed ground electric field is found to have a parabolically rising characteristic, concave upwards. However, the earlier simpler models do not predict most of the other characteristics.

Following are the main applications of the present work. The model developed can serve as a computer simulation tool for the lightning stepped leader and aid in the better analysis of the field data. A more accurate calculation of the attractive radius is possible with the present model as both charge distribution and stepwise propagating characteristics are deduced by coupling the electric field and air breakdown processes. In addition, as both permittivity and conductivities of the earth are considered, a more realistic estimation of the transient ground potential is now possible. Similarly a detailed description of the initial conditions for the simulation of the return stoke is now available.

\section{$7 \quad$ References}

1 BERGER K.: 'The earth flash' in GOLDE, R.H. (Ed.): 'Lightning, Part 1 - Physics of lightning' (Academic Press, 1977, 1st edn.)

2 UMAN, M.A., and KRIDER, E.P.: 'A review of natural lightning: Experimental data and modelling', IEEE Truns. Electromagn. Compat., 1982,EMC-24, (2), pp. 79-112

3 WAGNER, C.F., and HILEMAN, A.R.: 'The lightning stroke', AIEE Trans., 1958,36, pp. 229-242

4 LITTLE, P.F.: 'Transmission line representation of a lightning return stroke', J. Phys. D, Appl. Phys., 1978, 11, pp. 1893-1910

5 MATTOS, M.A. DA F., and CHRISTOPOULOS, C.: 'A nonlinear transmission line model of the lightning return stroke', IEEE Trans. Electromagn. Comput., 1988, EMC-30, (3), pp. 401406

6 HOOLE, P.R.P.: 'Modelling the lightning earth flash return stroke for studying its effects on engineering systems', IEEE Trans. Magn., 1993, 29, (2), pp. 1839-1844

7 BAUM, C.E. and BAKER, L.: 'Analytic return-stroke transmissionline model' in GARDNER, R.L. (Ed.): 'Lightning electromagnetics' (Hemisphere Publishing Corporation, New York, 1990,1st edn.)

8 DELLERA, L., and GARBAGNATI, E.: 'Lightning stroke simulation by the leader progression model, Part I: Description of the model and evaluation of exposure of free-standing structures', IEEE Trans. Power Deliv., 1990, 5, (5), pp. 2009-2022

9 THUM, P.C., LIEW, A.C., and WONG, C.M.: 'Computer simulation of the initial stages of the lightning protection mechanism', IEEE Truns. Power Appar. Syst., 1982, 101,(11), pp. 4370-4377

10 RIZK, F.A.M.: 'Modelling of lightning incidence to tall structure Part I: Theory', IEEE Trans. Power Deliv., 1994, 19, (1), pp. 162-170

11 BEASLEY, W.H., UMAN, M.A., and RUSTAN, P.L.: 'Electric fields preceding cloud-to-ground lightning flashes', J. Geophys. Res., $1982,87,(\mathrm{C} 7)$, pp. 48834902

12 UDAYA KUMAR, and NAGABHUSHANA, G.R.: 'Images in linear conducting dielectrics', IEE Proc., Sci. Meas. Technol. 1997, 144, (4), pp. 163-167

13 ALLIBONE, T.E.: 'The long spark' in GOLDE, R.H. (Ed.): 'Lightning, Part 1 - Physics of lightning' (Academic Press, 1977, 1st edn.)

14 HUTZLER, B., and HUTZLER-BARRE, D.: 'Leader propagation model for determination of switching surge flashover voltage of large air gaps', IEEE Trans. Power Appar. Syst., 1978, PAS-97, (4), pp. $1087-1096$

15 RIZK, F.A.M.: 'A model for switching leader inception and breakdown of long air-gaps', IEEE Trans. Power Deliv., 1989, 4, (1), pp. 596-606

16 UDAYA KUMAR, 'Electromagneticmodeling of the first stroke of a lightning flash'. PhD thesis, Indian Institute of Science, India, 1997

17 KUNHARDT, E.E., and LUESSEN, L.H.: 'Electrical breakdown and discharges in gases' (Plenum Press, 1983, 1st edn.)

18 OHTA, T., NAKANO, T., and MUROOKA, $Y$.: 'Electric field distribution in long-gap discharges'. Proceedings of 8th international symposium on High voltage engineering, Yokohama, Japan, August 1993,pp. 44.08

19 ALLEN, N.L., and GHAFF, A.: 'Temperatureand density effects on streamer propagation in air'. Proceedings of 8th international symposium on High voltage engineering, Yokohama, Japan, August 1993, pp. 40.02

20 GOLDE, R.H.: 'Lightning currents and related parameters' in GOLDE, R.H. (Ed.): 'Lightning, Part 1 - Physics of lightning' (Academic Press, 1977, 1stedn.)

21 UDAYA KUMAR, and NAGABHUSHANA, G.R.: 'A generalised definition for inductance and RLC type transient fields in coils'. Proceedings of 10th international symposium on High voltage engineering, Montreal, Canada, 25-29 Aug. 1997, Paper 3350

22 SHYY, W., CHEN, M.H., MITTAL, R., and UDAYKUMAR, H.S.: 'On the suppression of numerical oscillations using a non-linear filter', J. Comput. Phys., 1992, 102, pp. 49-62

23 GROVER, W.F.: 'Inductancecalculations' (Dover Publications, New York, 1962, 1st edn.)

24 UDAYA KUMAR, and NAGABHUSHANA, G.R.: 'A new model for the lightning stepped leader'. Proceedings of 10th international symposium on High voltage engineering. Montreal, Canada, 25-29 Aug. 1997, Paper 3351

25 SCHONLAND, L.H., HODGES, D.B., and COLLENS, H.: 'Progressive lightning V. A comparison of photographic and electrical studies of the discharge process', Proc. R Soc. Lond., 1938, CLXVI, pp. $56-75$

26 BAUM, C.E.: 'Properties of lightning-leader pulses' in GARDNER, R.L. (Ed.): 'Lightning electromagnetics' (Hemisphere Publishing Corporation, $N Y$, USA, 1992) 